\title{
Neuroeconomics and Management Educations: Perspectives for New Applications and Approaches
}

\author{
Sergei Titov ${ }^{1, *}$ and Evgeniy Pluzhnik ${ }^{1}$ \\ ${ }^{1}$ Moscow Technological Institute, 199334, Moscow, Russia
}

\begin{abstract}
Since the 1990s economics started importing interesting insights from psychology and the new discipline of behavioral economics elaborated many interesting models that changed the traditional economic and management theories. Nowadays the applications from neuroscience are extensively used in economics which led to the birth of neuroeconomics. The article attempts to overview the current state and topics in neuroscience and neuroeconomics in order to elaborate the suggestions pertaining to management education. The article is based on several examples of the neuroeconomic research and formulates new applications and approaches to management training curriculum and methods of learning.
\end{abstract}

\section{Introduction: Neuroscience in economics and pedagogy}

Both economic and learning activities imply that human brain not only actively participates in economic and education processes, but also governs human behavior in them. Modern neuroscience helps to understand what is going on in human brains, how internal mental processes can influence human skills and behaviors. However, classical economic and pedagogic theories are based on the presumption of the 'black box' which means that it is impossible to understand the internal mental processes in the human brain. Today this theoretical foundation needs significant updating by the results of neuroscientific research. The infusion of neuroscience in the economic theory led to the birth of neuroeconomics which tries to understand deeper the processes that are hidden in human heads but influence the economic behaviors of people [1]. Neuroeconomics shed the light on the brain mechanisms involved in the business and managerial activities [2]. The newly generated knowledge in neuroscience has been actively applying in pedagogy [3]. Hence, the insights from neuroeconomics can be used in the management education.

The article presented is structured as follows. In the first part, general techniques of neuroscience and its perspective direction of research are overviewed. In the second part, the most interesting areas of neuroeconomic research are covered. In the third part, several example of the neuroeconomic research are described and potential applications for management education are formulated. In the final part, all applications for management education are summarized and suggestions for the changes in curriculum and learning techniques are proposed.

\section{Neuroscience: its tools and purposes}

Neuroscience uses wide varieties of different tools which help not only define the functional areas of the human brain but also make conclusion about how the brain operates.

One of the widely used neuroscientific tools is brain imaging [4]. Brain imaging includes such technologies as electro-encephalograms, positron emission topography and functional magnetic resonance imaging. Brain imaging allows visual display of the human brain functioning during the execution of different tasks by testees.

Another neuroscientific technique is single-neuron measurement [5]. This technique implies that small electrodes are inserted into the brain and the experimenters obtain the opportunity to measure a single neuron's activation. Single-neuron measurement is restricted by and large to nonhumans due to the fact that the insertion of the electrodes damages the brains.

Another tool restricted primarily to animals is electrical brain simulation that uses electrical pulses to stimulate certain sites in the brain and generate new types of responses and behaviours [6].

The analysis of psychopathological states of brains and damaged conditions of brains can be also fruitful for neuroscientific research. Investigating people with mental diseases and disorders can help understand how the human brain operates in normal conditions [7].

Finally, traditional psychophysiological measurements like galvanic skin response, pupil dilation, heart rate, blood pressure can be used to analyze the processes in the human brain.

It is important to emphasize that neuroscience tries not only to map the internal functional structure of the human brain but first of all to understand how the brain

* Corresponding author: titov s@mti.edu.ru 
approaches different tasks, what the different processes occur in the brain, to which extent the human brain specializes the different processes in different areas.

\section{Neuroeconomics: Its purposes and applications}

Neuroeconomics can be understood as an intersection of economics and neuroscience which applies neuroscientific methods for deeper understanding and interpretation of real behaviour of economic agents [2]. The main topics in neuroeconomics include the study of preferences [8], utility and the rewarding system [9], influence of socioemotional entities of fairness and trust on decision making and interaction of economic agents [10], insights on leaning, memory and knowledge in organizational context [11] and the neural theory of decision making [12]. All these topics are very relevant to management and management education.

\section{Research in neuroeconomics: Examples and implications for management education}

\subsection{Reframing of organizational problems to utilize specialized automatic mental processes}

According to the modern neuroscience in the human brain there are not only cognitive and controlled processes but also automatic and affective processes. Automatic mental processes tend to be connected with the specialized areas of brain and be more effective because they are effortless and can be performed without participation of conciseness. Cosmides showed that the same intellectual problem if it is framed in terms that are less formal and more like the ordinary everyday situation can be solved by more people with less effort [13]. In the case of the formal description of the problem, the areas associated with the cognitive and controlled processes are activated.

Modern work force is more and more engaged in the intellectual knowledge-based operations. Hence, it is crucial more companies to make their knowledge workers perform tasks faster and in a more creative way. One of the lessons that can be inferred from the research by Cosmides is that the work of managers should be more connected with the reframing of the problems so that the workers can utilize better their automatic processes. However, nowadays management education underemphasizes this kind of managerial skills. Moreover, the managerial problem solving skills that are formed in business schools and universities are more oriented to more formal approaches to problems and pay little attention to the possible reframing of the organizational problems.

\subsection{Role of imagination in decision making}

The research on psychopaths with emotional deficits showed that these people have low capacity for imagination of different pictures with emotional impact on them [14]. They can correctly define of the basis of the pure rationality the best options that are given to them in the experiments. However, they are very indecisive in terms of choosing one of the options. On the cognitive level they fully understand what the most valuable option is. But they are not able to choose it because they lack emotions.

What can we infer from these results and transfer to the area of management and management education? It is not enough to calculate the value of the options. Managers should be able to create different images of different options. They need switch on their emotions, generate the positive image of the chosen option and communicate with other people not only on the language of numbers and formal logic but also on the language of images and emotions. Today management education accentuates the skills in mathematics and logics, the abilities to persuade people with facts, numbers, reason and common sense. However, managers also need to cultivate their emotional intelligence, imagination and persuasion with images of desirable future.

\subsection{Emotional intelligence and trust}

Very interesting findings were formulated during the neuroscientific monitoring of different participants of so called ultimatum game. This game implies that one person offers a part of a sum of money, usually $\$ 10$, to another person. The receiver or responder can accept or reject the offer from the proposer. If the receiver rejects the offer both participants receives nothing. If the offer is accepted by the receiver, both parties get according to the accepted offer.

According to game theory the rational response of the receiver should be to accept any positive sum of money, even if it is marginally higher than 0 . However, experiments with normal people playing the ultimatum game show that people's decisions are based primarily on emotional reaction rather than on pure reason. On average the proposer offers $40-50 \%$ of the sum and nearly $50 \%$ of receivers reject the options with less than $20 \%$ [15]. Success in the ultimatum game (and similar negotiation games) depends not on the logical skills, but on the ability to 'read minds' of the opponents, or in other words on ability to feel what the opponent considers as a just offer.

In the research by Hill and Sally the behaviour of normal and autistic people playing the ultimatum game was analyzed [16]. The researchers concluded that substantially more autistic people offered zero or the sum very close to zero. It is well known that autistic people are less emotionally intelligent and usually have difficulties in understanding emotions of other people. Autistic people tend to behave in an extremely rational way not paying attention to the expected feelings of other people. Normal people usually try to guess what other people can consider as fair in the ultimatum game.

McCabe and his colleagues found that people expressing more trust and cooperation in the different negotiation games more extensively activate the areas in 
brain that are connected with emotions [17]. Hence, human notions of fairness are deeply rooted in the emotional and not-controllable processes of the brain and they cannot be overridden by the cognitive and controlled mental processes associated with rationality.

In the research by Sanfey et al. the participants of the ultimatum game were studied with the help of fMRI technology [18]. The researcher found that the people facing apparently unfair offers activated the regions of the brain connected with the experience of negative emotions such as pain or disgust. These emotions seemed to be spontaneous. Despite the fact that other regions of the brains connected with planning and executive functions were activated also the behaviour of people was driven primarily by immediate emotional reaction.

Here again we can see how it is important for effective managers to develop their skills connected with emotional intelligence. Moreover, from the analysis of the ultimatum game from the neuroscientific perspective it is clear that managers should advance their ability to understand the potential emotional response to different decisions even if people try to hide it. Management education should not ignore that fact the perceptions of fairness on working places are real factors of effectiveness.

\subsection{Hormones and trust}

In the study by Zak et al. (2003) the researchers investigated the role of hormones in behaviour of people involved in a trust game [19]. The level of hormone oxytocin which usually rising during close social interactions (like breast-feeding or physical touching) was found to be extremely important. Oxitocin rose in people who really trusted their opponents in the game.

If the level of oxitocin can be increased by the demonstrating closer social interaction, managers should learn to use this physiopsychological mechanism in the situation where cooperation is crucial. The level of trust can be significantly increase by simple physical touching and showing interest to a person.

\subsection{Sleep-cycles and level of cooperation}

Gonzalez and Loewenstein showed that sleep-cycle can affect the level of cooperation in teams solving the same problem and in negotiations [20]. They found that 'night' people working in morning hours are statistically less cooperative than the same people working in evening hours. The same is true with 'morning' people. They demonstrate lower level of trust and cooperation while working in evening hours.

\section{Conclusions and discussions}

From the overview of the examples above we can suggest changes in the typical management education curriculum as well as in the methods of teaching managers. The neuroeconomics makes it clear that not only traditional courses should be included in the programs of management trainings but also advanced ones, such as:

- Creative problem framing,

- Imagination and creativity in management,

- Emotional intelligence and trust building,

- Neuropsychology of managerial job.

With regard to new teaching methods we recommend to use extensively group problem solving, provocative emotional clashes, mini business games, psychological experiments.

\section{References}

1. C.F. Camerer, G. Loewenstein, D. Prelec, Scand J of Economics 106 (3), 555-579 (2004)

2. P. Kenning, H. Plassmann, Brain Res Bull 67, 343354 (2005)

3. U. Goswami, Brit J Educ Psychol 74, 1-14 (2004)

4. Ph. Ball, Nature 412, 150-157 (2001)

5. A.A. Boulton, Neurophysiological Techniques: Applications to Neural Systems (Humana Press, 1990)

6. A. Selimbeyoglu, J. Parvizi, Front Hum Neurosci 46, 4 (2010)

7. A.R. Damasio, Descartes' Error: Emotion, Reason, and the Human Brain (G.P. Putnam, 1994)

8. M. Deppe, W. Schwindt, H. Kugel, H. Plassmann, P. Kenning,' J Neuroimagin 15, 171-182 (2005)

9. B. Knutson, R. Peterson, Games Econ Behav 25, 305-315 (2005)

10. A.G. Sanfey, R. Hastie, M.K. Colvin, J. Grafman, Neuropsychologia 41, 1218-1229 (2003)

11. A.G. Sanfey, J.D. Cohen, PNAS 101, 16709-16710 (2004)

12. P.W. Glimcher, A. Rustichini, Science 306, 447-451 (2004)

13. L. Cosmides, Cognition 31 (3), 187-276 (1989)

14. A. Bechare, A.R. Damasio, H. Damasio, S.W. Anderson, Cognition 50 (1-3), 7-15 (1994)

15. C. Camerer, G. Loewenstein, D. Prelec, J Econ Lit 43, 9-64 (2005)

16. E. Hill, D. Sally, Dilemmas and bargains: Autism, theory-of-mind, cooperation and fairness, (University College London, Working paper 2003)

17. K. McCabe, D. Houser, L. Ryan, V.L. Smith, T. Trouard 'A functional imaging study of cooperation in two-person reciprocal exchange', Proceedings of the NAS of the USA, 98 (20), 11832-11835 (2001)

18. A.G. Sanfey, J.K. Rilling, J.A. Aaronson, L.E. Nystrom, J.D. Cohen, Science 300 (5626), 1755-58 (2003)

19. P. Zak, R. Kurzban, W. Matzner Horm Behav 48, 522-527 (2005)

20. R. Gonzalez, G. Loewenstein, Effects of circadian rhythms on cooperation in an experimental game (Working paper 2004) 